\title{
$5 \mathrm{kHz}$ Thermometry in a Swirl-Stabilized Gas Turbine Model Combustor using Chirped Probe Pulse Femtosecond CARS. Part 2: Analysis of Swirl Flame Dynamics
}

\author{
Carson D. Slabaugh ${ }^{\mathrm{a}, *}$, Claresta N. Dennis $^{\mathrm{b}}$, Isaac Boxx $^{\mathrm{c}}$, Wolfgang Meier ${ }^{\mathrm{c}}$, Robert P. Lucht $^{\mathrm{b}}$ \\ ${ }^{a}$ School of Aeronautics and Astronautics, Purdue University, 701 W. Stadium Ave., West Lafayette, IN 47907-2045 \\ ${ }^{b}$ School of Mechanical Engineering, Purdue University, 585 Purdue Mall, West Lafayette, IN 47907-2088 \\ ${ }^{c}$ German Aerospace Center (DLR), Institute of Combustion Technology, Pfaffenwaldring 38-40, 70569 Stuttgart, Germany
}

\begin{abstract}
We have performed a detailed analysis of the temperature field in a turbulent swirl flame operating with a self-excited thermo-acoustic instability. The temperature field was measured using $5 \mathrm{kHz}$ chirpedprobe-pulse (CPP) femtosecond (fs) coherent anti-Stokes Raman scattering (CARS). The measurements are described in detail in the part 1 companion article. In this paper, part 2, a detailed analysis of the time-resolved temperature measurements and simultaneous pressure measurements is performed to provide insight into the dynamics and structure of the swirl-stabilized flame. This work is the first to capture the dynamics of the flame, flow, and coupled flow-flame processes using high-fidelity, spatially- and temporallyresolved thermometry in a flame of practical relevance. The time-averaged contour plot of the temperature field indicates that the flame is very flat and stabilizes approximately $10 \mathrm{~mm}$ downstream of the burner face. In this region, there are very significant temperature fluctuations indicating a very high level of unsteadiness. The temperature probability distribution functions (PDFs) are clearly bimodal in this region near the injector face. A Fourier analysis of the temperature time series revealed multiple coherent oscillatory modes. The strongest oscillation was found to be coherent and in-phase with an acoustic resonance at $314 \mathrm{~Hz}$, as expected from the Rayleigh criteria for the unstable flame. An analysis of the phase-conditioned average temperature fields show clearly an axial pumping of low-temperature reactants, which are consumed after a convective delay and result in a spike in the global heat-release rate. Continued analysis also revealed a 438 $\mathrm{Hz}$ oscillation that was found to correspond with the dynamics of convective transport by a helical precessing vortex core (PVC). The structure of the PVC, and its interaction with the flame, were studied based on the presence of this characteristic frequency in the power spectral densities computed throughout the flow. The precision and time-resolution of the CPP fsCARS measurements was also sufficient to enable computation of the integral time-scales as well as the PDFs of the temporal temperature gradients. A sample of state space trajectories were used to provide insight into the nature of coupling between the narrowband acoustic resonance and the broadband spectrum of turbulent flame processes.
\end{abstract}

Keywords: swirl flame, gas turbine, laser diagnostics, femtosecond CARS, thermometry 


\section{Introduction}

High power-density combustion technologies are designed to achieve flow characteristics that support sustained, elevated rates of chemical heat release. Often, swirling flows are used in such designs, as they effectively promote turbulent transport and enhance mixing processes that lead to rapid progress of chemical reactions and a compact flame shape. Beyond a critical level of swirl, vortex breakdown results in the formation of the central recirculation bubble (CRB), which transports heat and active chemical species from the combustion product gases back to the flame root. This central aerodynamic blockage serves as a self-sustaining ignition source, stabilizing the high-power flame and supporting increased levels of reactant mass consumption [1-4].

The hydrodynamic structure of swirling flows is three-dimensional and unsteady, with multiple large-scale turbulent structures interacting over a broad spectrum of spatial and temporal scales. With combustion present, chemical reactions modify the behavior of the flow through spatio-temporal evolution of the thermochemical state and thermo-physical properties. Under certain conditions, the local interaction of turbulence and chemistry with the resonant acoustic field can also give rise to global instabilities. Fundamental understanding of these processes is necessary to advance combustion technologies and avoid the harmful impacts of such conditions [5-7]. One strategy to study these effects has been to develop laboratory-scale, gas turbine model combustors (GTMCs) that represent the flow structure, geometry, and other complexities found in systems of practical relevance [8-12]. As such, the Dual-Swirl GTMC (developed at the German Aerospace Center, DLR) has become a widely-used platform for detailed experimental and numerical investigations of these very important flows.

Weigand et al. [13] and Meier et al. [14] performed the first detailed characterization of this burner. They studied three flame conditions: one which burned stably (Flame A), one with pronounced self-excited thermoacoustic pulsations (Flame B), and a third operating near the lean blow-out limit (Flame C). Utilizing laser Doppler velocimetry (LDV), laser Raman scattering, and planar laser induced fluorescence (PLIF) imaging of hydroxyl $(\mathrm{OH})$ and methylidine $(\mathrm{CH})$, they reported the time-averaged flow and flame structure as well as a statistical analysis of the planar distributions of temperature, species concentrations, and mixture fraction. It was found that, despite having very similar time-averaged velocity fields, each flame showed pronounced differences in the structure of the reaction zone due to changes in unsteady mixing and finiterate progress of chemical reactions. In all cases, the flames were lifted, stabilizing approximately $10 \mathrm{~mm}$ downstream of the fuel injection plane. Within the CRB, the gas mixtures were almost completely reacted, with a thermo-chemical state very close to equilibrium. Partially-reacted mixtures were detected within the Inner Shear Layer (ISL) formed between the fresh gas injection jets and the CRB, where the measurements

\footnotetext{
* Corresponding author

Email address: cslabau@purdue.edu (Carson D. Slabaugh )
} 

unstable condition (Flame B), additional periodic oscillations in the velocity field led to enhanced mixing effects between the reactants and hot gases within the CRB. This resulted in the rapid progress of chemical reactions and significantly more compact flame structure.

To improve understanding of the coupled mechanisms underpinning the thermo-acoustic oscillation observed in Flame B, Meier et al. [15] acquired a phase-locked measurement set which captured the periodic fluctuations in heat release (estimated by $\mathrm{OH}^{*}$ chemiluminescence), flow velocity, mixture fraction, and fuel distribution occurring at the known oscillation frequency. Fluctuations in pressure and heat release were found to be nearly in-phase, as expected from the Rayleigh criterion. The combustion chamber pressure oscillation resulted in phase-dependent variations in the inflow velocity and, consequently, the reactant mass flow rate. Phase-locked laser Raman measurements revealed that the periodic reactant inflow was correlated with a phase-dependent equivalence ratio oscillation, likely due to mismatched impedances in the fuel and oxidizer supply manifolds. Evidently, the periodic inflow oscillation of fuel-rich mixture was found to have a direct phase relationship with the heat release oscillations, corresponding to the convective time delay from the nozzle to the reaction zone.

Planar particle image velocimetry (PIV) and scalar field imaging (specifically, planar laser induced fluorescence, or PLIF) measurements were then performed to reveal the spatial structure of the unstable flame and the combustor flow-field. Sadanandan et al. [16] used both techniques, simultaneously, to study the structural coupling of flow-flame interactions. The measurements revealed vortices within the Inner Shear Layer (ISL) that were staggered across the flow centerline with increasing distance from the nozzle exit. Stohr et al. [17] continued to study these structures and correlated their presence to a single, large scale coherent turbulent structure; a helical Precessing Vortex Core (PVC). Residing within the ISL, the PVC was found to enhance transport of burned and unburned gases within the spatially developing mixing layer, thus promoting flame stabilization through entrainment of reactants with high-temperature gas.

In recent years, the rapid development and commercial availability of high-repetition-rate, diode-pumped solid-state (DPSS) lasers with short pulse durations has made temporally-resolved planar measurements in laboratory-scale flames an accessible option. The simultaneous integration of these systems has supported concurrent velocity field and (qualitative) scalar field measurements at $\mathrm{kHz}$ interrogation frequencies to yield greater understanding of the space-time evolution of flow-flame interactions [18-23]. Measurements in the Dual-Swirl GTMC have shown that heat release takes place, primarily, in two interconnected regions of the flow: 1) at the lower stagnation point of the central recirculation bubble and 2) in a helical zone coupled with the presence of the precessing vortex core within the inner shear layer. The influence of the PVC on flame stabilization is attributed to low strain rates and enhanced mixing of burned and fresh gases (due to large scale transport). Fluctuations in the position or size of the CRB were found to result in an enmeshed motion of the reaction zone while the flame remains, locally, within the low-velocity regions of the ISL. The 
flame dynamics are coupled to the CRB in that the stagnation point serves as the leading point of ignition for the reactions within the ISL [24-28].

As a planar technique, PLIF can provide detailed spatial information about the structure of the flame. However, it does not yield quantitative information with respect to the thermo-chemical state of the flow. Linear scattering techniques, such as laser Raman and Rayleigh scattering, can provide detailed, quantitative, single-shot measurements of many gas properties, including temperature and major species concentrations. However, the signal generated by these processes is very weak, hence, the measurements require very high laser fluence and have, historically, been limited to low-bandwidth (single-shot) detection. In the case of Rayleigh scattering, the Rayleigh cross-section assumptions required in data processing also limit the applicability of the technique to clean flames with tailored fuels.

Coherent anti-Stokes Raman scattering (CARS) is a third-order, nonlinear spectroscopic technique that can also provide high-fidelity measurements of temperature, species concentration, and other flow properties. The signal generated by the CARS process is a coherent beam emitted from the probe volume. This unique aspect of the CARS technique makes it suitable for application in hostile flame environments where the signal can be efficiently collected with spatial, spectral, and polarization filtering. The CARS technique does not require tailored reactants, making it a robust tool for measurements in highly unsteady, practical reacting flows [29-39]. In recent years, there has been considerable progress in the application of high-peak power, ultra-fast laser sources for high-fidelity CARS thermometry at interrogation frequencies sufficient to time-resolve the large-scale, dynamic processes in turbulent flames [40-48].

In this study, ultrafast CARS temperature measurements were performed in the DLR Dual Swirl GTMC operating with high-amplitude, self-excited thermo-acoustic oscillations. As fully-described by Dennis et al. (part 1 of this work) [49], the measurements were shown to have excellent accuracy and precision. A comparison of time-averaged statistics computed from the chirped-probe-pulse (CPP) femtosecond (fs) CARS measurements and laser Raman measurements performed at DLR [14, 15], also showed good agreement. The objective of this effort (part 2) is to report the utilization these unique new measurements in facilitation of an improved understanding of highly-unsteady swirl flame dynamics.

\section{Experimental Configuration}

The DLR Dual-Swirl GTMC has been the subject of multiple previous studies in which the geometry is described in detail $[13-15,27]$. The analysis performed in this work is based on the measurements reported in Dennis et al. [48, 49], where the CCP-fsCARS system is also rigorously described. Hence, only a brief summary of the flame and measurement systems is provided herein.

\subsection{Burner and Flames}

A schematic diagram of the DLR Dual-Swirl burner is shown in Figure 1. Dry air is supplied to a plenum through an acoustic isolation section with a choked orifice, upstream. Two radial swirlers impart 
an azimuthal velocity component to the flow before it is introduced to the combustion chamber. Fuel is injected, without swirl, through an annular ring of channels between the central and outer co-swirling air supplies. The exit plane of the fuel and central air supplies is $4.5 \mathrm{~mm}$ upstream of the combustion chamber inlet plane. The flame is contained within an optically-accessible combustion chamber with fused quartz windows forming an $85 \mathrm{~mm}$ square cross section that is $110 \mathrm{~mm}$ in length. The exhaust plate contains a conical contraction which transitions to a $40 \mathrm{~mm}$ diameter exhaust tube.

[Figure 1 about here.]

In this study, the flame was operated at the condition previously defined as Flame $B$ [13, 14]. Fuel and oxidizer were supplied to the combustor at rates of $12.3 \mathrm{~g} / \mathrm{min}$ and $281 \mathrm{~g} / \mathrm{min}$, respectively. The fuel was chemically-pure methane gas and the oxidizer was dry, compressed air. Both flows were supplied through electromechanical mass flow controllers (Porter Series 200) at ambient temperature and the combustor was operated at atmospheric pressure. The global equivalence ratio was 0.75 , resulting in a flame thermal power of $10 \mathrm{~kW}$ and a computed global adiabatic flame temperature of $1915 \mathrm{~K}$. The Reynolds number of the total incoming reactant flow, based on the $25 \mathrm{~mm}$ nozzle exit diameter, was approximately 15000 . The swirl number (S) was computed based on nonreacting LDV measurements performed by Weigand et al. [13] to be 0.55 , where $S=\left(\int_{0}^{R} w u r^{2} d r\right) /\left(R \int_{0}^{R} u^{2} r d r\right), R$ is the nozzle exit radius, and $u$ and $w$ are the axial and azimuthal components of velocity, respectively. It should be noted that slight differences in the structure and dynamics of the flame were observed when operating the burner at Purdue, compared to previous measurements performed at DLR. As discussed in the companion article (part 1), this flame is highlysensitive to boundary conditions. Consequently, it is thought that these changes in flame behavior are the result of uncertainty in the ancillary experimental systems and ambient conditions of the two laboratories.

Prior to data acquisition, the burner was allowed to thermally stabilize for thirty minutes. A microphone (Bruel and Kjaer, Type 4939) was installed in the upstream plenum to monitor the chamber acoustics. The frequency response of the transducer covered a range from $4 \mathrm{~Hz}$ to $100 \mathrm{kHz}$. The microphone signal was recorded, simultaneously, with the laser system trigger and the EMCCD gate signal at a sampling frequency of $100 \mathrm{kHz}$ using a high-speed, analog-to-digital data acquisition system (National Instruments 16-bit PXIe-6366). Fourier transformation of the pressure signal revealed a narrowband frequency peak at $314 \mathrm{~Hz}$; known to correspond with large-amplitude thermo-acoustic pulsations. It was previously shown that the combustion chamber pressure dynamics are well-captured by the plenum microphone (with lower signal noise) after imposing a constant phase shift of the dominant acoustic frequency [15, 24]. Hence, the plenum microphone signal was used to identify the phase angle of the acoustic pulsation, at the nozzle exit, corresponding to each CARS temperature measurement. 


\subsection{CPP-fsCARS System}

A schematic diagram of the laser system used to perform chirped-probe-pulse femtosecond CARS (CPPfsCARS) measurements is shown in Figure 2. The fundamental output of the Ti:Sapphire oscillator and regenerative amplifier (Coherent, Legend Elite Duo) provided $2.0 \mathrm{~mJ} /$ pulse of $800 \mathrm{~nm}$ light with a $60 \mathrm{fs}$ (FWHM) pulse duration at a repetition rate of $5 k H z$. The beam was split (90/10) to pump an optical parametric amplifier (OPA) with a frequency doubled output centered at $675 \mathrm{~nm}$ to generate the pump pulse. The remaining $10 \%$ of $800 \mathrm{~nm}$ light was used for the Stokes and probe pulses (6\% and $4 \%$, respectively) to target the Raman resonance of nitrogen near $2330 \mathrm{~cm}^{-1}$ with the nearly Fourier transform limited pulses. The probe pulse was directed through a dispersive rod, $30 \mathrm{~cm}$ in length, to induce chirp, with the long wavelength components arriving at the probe volume first. In this manner, the temporal decay of the Raman coherence was mapped into the frequency domain. The CPP-fsCARS signal was recorded using a spectrometer $(0.25 \mathrm{~m}$ with a $1200 \mathrm{~g} / \mathrm{mm}$ grating) and an EMCCD camera (Andor iXon) over $1 \mathrm{~s}$ sampling times (5000 laser shots). The beams were overlapped in a folded BOXCARS phase-matching geometry, which restricted the measurement probe locations to $>5 \mathrm{~mm}$ from any surface. The spatial resolution of the probe volume was $400 \mu \mathrm{m}$ by $50 \mu \mathrm{m}$ (FWHM) in the direction of beam propagation and beam-normal directions, respectively [48].

[Figure 2 about here.]

The time-averaged flow field of Flame B was measured by Stöhr et al. [16, 27] and is shown in Figure 3A with the CPP-fsCARS measurement locations overlaid. The streamlines, colored by the three-component velocity magnitude, illustrate the presence of a high-velocity (annular) reactant jet inflow, accompanied by an inner and outer recirculation zone. As previously discussed, time-resolved planar measurements (PIV and PLIF) have shown that the interaction between these structures, particularly within the inner shear-layer, is highly unsteady (see Figure 3B). The spatio-temporal evolution of the flow instabilities is strongly coupled to the structure and dynamics of the flame. Consequently, the spatial density of the CPP-fsCARS probe locations was increased in this region.

[Figure 3 about here.]

\section{Results and Discussion}

The proceeding analysis was developed from the dataset reported in part 1 of this work [49], wherein CPPfsCARS temperature measurements were shown to have very high accuracy and precision $( \pm 3 \%$ and $\pm 2 \%$, respectively) in the highly-turbulent, unstable swirl flame. A minimum of 5000 single-shot measurements were collected at each grid location over $1 s$ sampling intervals to achieve well-converged statistics, even with phase-conditioning. During each test, twelve random measurement locations were probed for five $1 s$ intervals 
to verify repeatability and convergence of the data. Through these comparisons, it was found that there was no considerable variation in first or second-order statistics computed from each of the independently sampled datasets. Convergence of the first-order moments was typically achieved within 1000 measurements, resulting in a mean standard error $\left(S E M=T_{r m s}^{\prime} / \sqrt{N}\right)$ of $<10 K$ throughout the flow-field. The SEM was $<5 K$ for large portions of the domain when the full 5000 samples were used. Convergence of second-order moments was achieved to $\leq 20 \mathrm{~K}$ with an average of 293 samples, while 600 samples improved convergence to $<10 K$ for a majority of the probe locations.

The temperature spectra presented in this section were computed with several window sizes and offsets to determine a balance between convergence and spectral resolution. The reported spectra are an ensembleaverage of five sub-sampled data series, 800 samples in length. Between each data segment, 200 samples of data were skipped to ensure independence. To verify the processing methods, the fluctuation magnitude was computed over a narrow frequency band by integration under the PSD curve. This value was then compared with the measured fluctuation magnitude of the time series, digitally-filtered over the same frequency band. Agreement was typically within $\pm 10 \%$ using a standard bandwidth described by the FWHM of the PSD peak.

\subsection{Time-Averaged Statistics}

[Figure 4 about here.]

To begin, Figure 4A presents the time-averaged temperature field computed from 5000 measurements at each probe location. Indicated by the black circles, the CARS measurements were acquired at a series of discrete points in the flow. The continuous temperature field presented in Figure 4A was generated with a linear interpolation in space (between the discete measurement locations) and is represented on a continuously-varying colormap index for plotting purposes only; no analysis was performed on the interpolated fields. Figure 4 shows that the reaction zone is very compact, with cold gas (reactant) penetration extending $<10 \mathrm{~mm}$ from the nozzle exit and peak temperatures appearing by $y=20 \mathrm{~mm}$. Downstream of the reaction zone, and near the flow centerline, the measured temperatures are reduced as a result of convective and radiative heat loss from the fully-reacted, high-temperature gas through the windows of the optical chamber. The zone of intermediate gas temperatures corresponds, approximately, with the location of the base of the CRB (along the centerline) and the concomitant ISL [17, 25-27].

[Figure 5 about here.]

Rapid progress of chemical reactions and highly-unsteady fluid mechanical processes lead to a fast homogenization of the temperature field, as indicated by the flat radial temperature distribution $15 \mathrm{~mm}$ downstream of the nozzle exit. The root-mean-square (RMS) temperature fluctuation field is shown in Figure 4B, with extracted radial profiles (Figure 5B). These figures show that the time-averaged, peak fluctuation amplitude 
occurs at $y<15 \mathrm{~mm}$, which, as expected, corresponds to the structure of the intermediate temperature zone captured in the time-averaged field (Figure 4A).

Figure 6 presents probability distribution functions (PDFs) at select grid locations along the flow centerline (through the stagnation region) and at $x=15 \mathrm{~mm}$ (through the ISL). The PDFs are arranged in two columns based on their $x$ position, with each respective legend indicating the probe location, time-averaged mean, and fluctuation RMS. At a height of $y=5 \mathrm{~mm}$, the sample distribution is very broad, with temperatures varying from the ambient incoming reactant temperature to slightly greater than the global adiabatic flame temperature. This is expected with instantaneous measurements in a non-premixed flame, where local fuel-rich mixtures will produce fully-reacted temperatures that are greater than the computed equilibrium temperature for the globally lean mixture. The probability distributions are broad throughout the flow for $y \leq 15 \mathrm{~mm}$, then narrow considerably for $(y>30 \mathrm{~mm})$. At $(x, y)=(15,30) \mathrm{mm}$, the mean temperature is within $25 \mathrm{~K}$ of the computed adiabatic flame temperature. In the post-flame gases, including the CRB, the width of the distribution is significantly reduced, with fluctuation RMS values around $200 K$ and a peak probability centered approximately $120 \mathrm{~K}$ below $T_{A F}$.

[Figure 6 about here.]

There is a consistent bimodal shape to the PDFs for probe locations where $y \leq 15 \mathrm{~mm}$. The low temperature peaks correspond to samples where the gas within the probe volume is composed primarily of unburned reactants while the highest temperatures indicate the presence of a fully-reacted gas mixture. With increasing height, the low temperature peak remains fairly narrow, while the peak location is shifted to increasing temperatures as a result of mixing effects between burned and unburned gases (prior to ignition). With increasing height, the integral probability of the low temperature peak decreases as chemical reactions progress. Correspondingly, the strength of the broad, high-temperature group increases, as expected from the previous analysis of the time-averaged fields (Figure 4). Coupled with a high-probability of intermediate temperatures, the broad nature of the high temperature peak is indicative of highly-unsteady fluid mechanics and finite-rate chemistry effects. Utilizing the high precision and temporal bandwidth of the CPP-fsCARS dataset, deconvolution of these processes is the principal focus of the remainder of this work.

\subsection{Thermo-Acoustic Pulsations}

Figure $7(\mathrm{~A})$ presents a spectrogram of the microphone signal, corresponding to the combustion chamber pressure at the plane of nozzle exit. The spectrogram was computed from the signal fluctuation relative to the time-averaged mean, where $f(t)=\langle f\rangle+f^{\prime}(t)$. The chosen temporal envelope was $100 \mathrm{~ms}$, with $50 \mathrm{~ms}$ of overlap. There is a high-degree of stability in the dynamic signal, with one clearly defined frequency over the entire length of data acquisition (indicated by the horizontal line). This high-intensity peak was consistent throughout the acoustic measurements at each CARS probe location, indicating the presence of a 

$8 \mathrm{~A}$ shows the accompanying power spectral density computed from the temperature measurement time series acquired at $(x, y)=(15,5) \mathrm{mm}$. Plotted on a semilog scale, the strongest mode of the temperature oscillations is found at $314 \mathrm{~Hz}$. All power spectra presented in this work were computed using Welch's method [50].

[Figure 7 about here.]

The cross-coherence quantifies the level of frequency-domain correlation as a ratio of the power-spectral energies of two time-series and provides a measure of system coupling (Figure 8B). It is computed using equation 1 ,

$$
C_{a b}=\left|P_{a b}(f)^{2}\right| /\left(P_{a a}(f) P_{b b}(f)\right)
$$

where $P_{a a}$ and $P_{b b}$ are the auto-spectra of signals $a$ and $b$, respectively, and $P_{a b}$ is their cross-spectrum. Figure $8 \mathrm{~B}$ shows, mathematically, that the $314 \mathrm{~Hz}$ oscillations in temperature and pressure are strongly coupled. The cross-spectrum phase further reveals that the lag between these coherent dynamic processes remained approximately zero throughout the near-field region, as expected from the Rayleigh criteria for a flame operating with self-excited, thermo-acoustic oscillations.

[Figure 8 about here.]

Sampled, simultaneously, at a sufficiently high frequency, the CPP-fsCARS temperature measurements can be conditioned with respect to the stable, cross-coherent chamber pressure fluctuation at $314 \mathrm{~Hz}$. For this analysis, each temperature measurement was assigned to the nearest of eight discrete phase angles, beginning with the peak in the chamber pressure (as shown in Figure 9). The phase-conditioned mean temperature fields corresponding to each discrete phase angle of the coherent oscillation are shown in Figure 10. Again, the continuous temperature field was computed using a linear interpolation in space and is represented on a continuously-varying colormap index (similar to figure 4), for visualization purposes only. A discrete phase-angle resolution of $\pi / 4$ was chosen because it maintained good convergence $(S E M<8 K)$ of the phase-conditioned statistics computed at each probe location.

[Figure 9 about here.]

[Figure 10 about here.]

At the start of the cycle, the chamber pressure is at a maximum and the axial penetration of the fresh reactants (cold gases, $T<600 K$ ) is roughly at a minimum. This is because the driving pressure difference $(\Delta P)$ between the reactant supply manifolds and the combustion chamber is also at a minimum. 

reactants are consumed and the flame propagates, axially, upstream. As the cycle progresses, the chamber pressure drops ( $\Delta P$ increases) and a surge of fresh reactants is observed, beginning at a phase angle of 4 and continuing through 6 . From phase angles 7-8 and returning to 1-2, the cold gases are mixed and consumed by the flame, resulting in a large number probe locations having intermediate temperatures, as observed in Figure 10.

The observed cyclic pumping and consumption of fresh gas is consistent with the findings of Meier et al. [15], where phase-locked laser Raman measurements revealed an acoustically phase-dependent modulation in the fuel mass flux into the combustion chamber. After a convective time-delay, the resulting equivalence ratio oscillations led to an increase in heat-release amplitude that was thought to couple with the resonant acoustic field of the chamber. The time-resolved CPP-fsCARS measurements have confirmed this mechanism of thermo-acoustic coupling, through direct computation of the $C_{T, P}$ coherence, cross-coherent phase angle, and the corresponding phase-conditioned temperature fields.

Downstream, coupling of the resonant acoustic field with global heat release dynamics resulted in periodic oscillations of bulk, post-flame gas temperature. Shown in Figure 11 (A), the phase-conditioned mean temperature profiles remain in good agreement, throughout the cycle, as a function of distance from the centerline. Varying approximately $150 \mathrm{~K}$ in amplitude, the peak temperatures of the downstream gases are shown to correspond with phase angles 7,8 , and 1 . The temperature peak is coupled with the peak in chamber pressure.

\section{[Figure 11 about here.]}

The RMS of the temperature fluctuation relative to the phase-conditioned average $\left(T(t)=\left\langle T\left(\phi_{T A}\right)\right\rangle+\right.$ $\left.T^{\prime}\left(\phi_{T A}, t\right)\right)$ indicates that the highest amplitude fluctuations in temperature (aside from those occurring at $314 \mathrm{~Hz}$ ) correspond to the chamber pressure minimum. Beyond this observation, however, the structure of the radial profiles is much more difficult to decipher. While a strong phase-dependence is observed in the temperature fluctuations relative to the phase-conditioned mean (particularly from $x=5-20 \mathrm{~mm}$ ), the overall $T_{R M S}^{\prime}$ amplitude remains quite high. This indicates the presence of other dynamic processes that are not occurring at $314 \mathrm{~Hz}$, and, hence, remain unresolved in this analysis. The power spectrum of the temperature fluctuations relative to the phase-conditioned mean is shown in Figure 12 (red). Plotted against the original power spectrum of the temperature fluctuations relative to the time-averaged mean (blue, shown previously in Figure 8A), it is seen that the phase-conditioned process effectively isolated only the narrowband periodic oscillation at $314 \mathrm{~Hz}$. The remaining coherent signal content has no direct phase relationship to the single-mode averaging process. Hence, the remaining fluctuations will be effectively averaged over all random phase angles along with the stochastic content of the signal.

[Figure 12 about here.] 


\subsection{Hydrodynamic Processes}

To further delineate the spatio-temporal dynamics of the unsteady temperature field, Figure 13 presents the power spectral densities computed at each probe location across the burner radius for fixed distances from the nozzle exit. Throughout the flame zone (roughly, $y \leq 15 \mathrm{~mm}$ ), four distinct peaks are observed: one which corresponds to the thermo-acoustic pulsation at $314 \mathrm{~Hz}$, and three additional frequencies at $438 \mathrm{~Hz}$, at $124 \mathrm{~Hz}$, and (albeit weak) at $752 \mathrm{~Hz}$. Through comparison with an extensive database of complementary measurements performed at the same flame conditions, it was possible to correlate one of the secondary peaks with the presence of a helical precessing vortex core (PVC) [2, 3, 17, 21, 24, 27]. Residing within the inner shear layer (ISL), the instantaneous velocity field, shown in Figure 3B, indicates a pair of vortices in axially staggered locations across the flow centerline, consistent with the structure of the PVC. Through the detailed analyses reported in Meier et al. [15] and Steinberg et al. [51], the dynamics of this PVC are well-known such that the frequency at which the PVC circumscribes the flow centerline scales linearly with the inlet bulk velocity at a fixed Strouhal number of 1.35 , where $S t=\left(f_{P V C} * d_{n o z z l e}\right) / u_{x, b u l k}$. The predicted precession frequency is $441 \mathrm{~Hz}$, which very closely agrees with the $438 \mathrm{~Hz}$ oscillation measured in this dataset (see Figure 14). Consequently, the tertiary peaks observed at $124 \mathrm{~Hz}$ and $752 \mathrm{~Hz}$ can be identified as the sum and difference frequencies between the thermo-acoustic instability and the helical vortex core precession. Representing the interaction of these two dynamic processes, the time-resolved CPP-fsCARS measurements are the first to capture, quantitatively, this nonlinear coupling in the Dual-Swirl Burner temperature field.

[Figure 13 about here.]

[Figure 14 about here.]

The spatial structure of these dynamic flow processes is elucidated in Figure 13. Beginning at $y=5 \mathrm{~mm}$, there is roughly equal power in the oscillations at $314 \mathrm{~Hz}, 438 \mathrm{~Hz}$, and $124 \mathrm{~Hz}$ at the burner centerline, which corresponds with the time-averaged spatial location of the stagnation point. Immediately off-center, the strength of the Fourier modes associated with the PVC (at $438 \mathrm{~Hz}$ ) and the PVC-TA difference frequency (at $124 \mathrm{~Hz}$ ) increase by a factor of five and remain strong moving radially-outwards through the inner shear layer (see Figure 3) while the modes associated with the thermo-acoustic frequency remain nearly constant and at low power (for $x \lesssim 10 \mathrm{~mm}$ ). Utilizing phase-resolved, three-component LDV, Weigand et al. [13] reported that the $\mathrm{CRB}$ varied in size over the course of the thermo-acoustic cycle, resembling an axial pumping motion (corroborated by the phase-conditioned, mean temperature fields presented in this work). Translation and modulation of the location and size of the CRB forces a coupled response of the ISL, where the PVC resides. Hence, there is a coupled, global motion of the reaction zone due to the axial translation of the CRB, while it remains locally enmeshed with the circumscription of the helical vortex core [17]. In effect, the axial (helical) extension and compression of the PVC, displaced by the breathing CRB, provides indication of the boundary of the PVC through the relative amplitudes of the $124 \mathrm{~Hz}$ and $438 \mathrm{~Hz}$ modes. 
[Figure 15 about here.]

[Figure 16 about here.] 
Figure 17 shows the PDFs of temporal temperature gradients at the centerline $(\mathrm{A})$ and at $x=15 \mathrm{~mm}(\mathrm{~B})$ for a series of burner heights. The gradients were normalized by the adiabatic equilibrium flame temperature $\left(T_{A F}\right)$ divided by the integral time-scale $\left(t_{0}\right)$ computed from the temperature time series at that specific probe location. At both $x$ locations, the shapes of the PDFs are very similar for heights of $5 \mathrm{~mm}$ and $10 \mathrm{~mm}$ above the nozzle exit, which is reasonable to expect based on the flat reaction zone observed in the time-averaged temperature field (Figure 4). The slight departure at $(x, y)=(0,10)$ could be due to improved resolution of $t_{0}$ near the flow centerline and with increasing height. For $y \geq 15 \mathrm{~mm}$, the PDFs become significantly more narrow and show an exponential scaling (which appears as a straight line on a loglinear plot). Exponential scaling has been previously reported in non-reacting flows due to the intermittent nature of the scalar dissipation fluctuations $[52,53]$. This is thought to further confirm that there is a significantly-reduced propensity for chemical reactions to occur at these locations. Rather, the temperature field fluctuations for $y \geq 15 \mathrm{~mm}$ are evidence of hydrodynamic and thermal mixing processes in the intensely turbulent flow.

\section{[Figure 17 about here.]}

\subsection{Nonlinear Dynamics}

The state-space trajectory of a time-resolved dataset can provide a physically-tractable representation of dynamic signal behavior in terms of energy conservation. As a discrete analog to phase-space analyses of continuous functions, Figures 18-19 present the state-space dynamics of the measured pressure and temperature fluctuations (relative to the time-averaged mean) and their respective temporal derivatives. Each time series is mean-subtracted and normalized by the peak fluctuation amplitude (indicated by the tilde), and the temporal derivative was computed using a central difference scheme (a single-sided difference was used at the start and end of the time series, but these points were subsequently truncated before further analysis). In a state-space, any closed trajectory contains an equilibrium condition of the system. The amplitude of the orbit radius relative to the equilibrium point gives an indication of the oscillation strength, while the angular velocity contains information about the frequency [54].

[Figure 18 about here.]

[Figure 19 about here.]

Figure 18 presents the evolution of the chamber acoustic measurements. The first important observation is the absence of bifurcations (or jumps) in the state-space trajectory of the pressure field dynamics. Furthermore, the orbit radius does not show any steady growth or decay, but rather maintains a stable radius about the neutral stability point (here, the time-averaged mean). Thus, the system is both driven and damped; exhibiting limit cycle behavior, which reinforces the notion of stability in the self-excitation 
process that was previously determined by the cross-coherent phase. Hence, this dynamic equilibrium is achieved as a result of the periodic addition of energy back into the acoustic wave at a locked phase angle corresponding to the angular frequency of the state-space orbit.

The state-space of the corresponding temperature time-series, measured at $(x, y)=(15,5) \mathrm{mm}$, is shown in Figure 19A. In distinct contrast to the microphone signal, this highly-intertwined trajectory underscores the broadband nature of the coupled turbulence-chemistry-acoustic interactions within the reaction zone. With increasing height, the temperature field dynamics become more discernible as state-space orbits of the multi-scale dynamic processes stratify into distinct orbits. In Figure 19B, a dense cluster of state realizations is present $0<\widetilde{T^{\prime}}<0.5$ and $\widetilde{\delta T / \delta t} \approx 0$. A periodic orbit is revealed, emanating from the cluster and traversing a roughly circular trajectory with an angular frequency $\omega \approx 2 \pi /(16 * 0.0002 s)$, corresponding very nearly to the frequency of the thermo-acoustic pulsation at $\omega / 2 \pi \approx 312 \mathrm{~Hz}$. At a height of $30 \mathrm{~mm}$, the density of the clustered group increases, while the radius and angular frequency of the larger periodic orbit remains intact. Even within the structure of the dense cluster, the effect of the global heat release oscillation remains clear, with similar low-amplitude state-space orbits occurring at an angular frequency that remains consistent with the thermo-acoustic oscillation. The orbit radius bifurcations at $y=15 \mathrm{~mm}$ and at $y=30 \mathrm{~mm}$ indicate that the temperature fluctuations corresponding to the thermo-acoustic frequency are the result of two distinct processes. The large-radius orbits indicate the presence of heat release occurring within the measurement probe volume while low-amplitude orbits are indicative of periodic oscillations in the post-flame gas temperature.

Comparison of Figures 18 and 19 highlights the fundamental challenge to understanding and mitigation of combustion instabilities in practical combustors, such as the Dual Swirl GTMC, where narrowband frequency coupling between the acoustic resonance and broadband spectrum of chemical and turbulent processes can completely alter the behavior of the flame. Despite the seemingly intractable evolution of the temperature time-series near the nozzle exit (Figure 19A), it is possible to fully-describe these trajectories as a series of orbits about equilibrium stability points. While the identification and characterization of these points is beyond the scope of this work, an alternative method to broadly characterize these nonlinear dynamic processes is through data-based chaos analysis. Broadly defined, chaotic behavior is characterized by aperiodic, long-term dynamics of a bounded, deterministic system that exhibits sensitive dependence on initial conditions. The spectrum of Lyapunov exponents can be computed to determine the level of chaos; providing a measure of the rate of separation of two state-space trajectories that begin near the same initial condition, or an average stretching of the space. The sign of the Lyapunov exponent signifies the presence of chaos and the magnitude measures the level of chaotic behavior. Specifically, a bounded dynamical system with a positive Lyapunov exponent is chaotic, and the value describes the average rate at which predictability is lost. A negative value implies a fixed state or a periodic cycle [54-64]

Figure 20 presents a contour map of the largest Lyapunov exponent computed from the spectrum at each 

strong chaotic behavior through the flame zone, where finite-rate chemistry and turbulent mixing processes control highly-nonlinear flow-flame interactions coupled to the global impact of thermo-acoustic pulsations. The location of the transition from a positive to a negative largest Lyapunov exponent, $\left.(x, y)\right|_{\Lambda_{1}=0}$, bounds the reaction zone with a trajectory and height that agree well with the other analyses presented in this work. Downstream of the flame zone, $\Lambda_{1}<0$, which is expected as the flow is fully-reacted in this region. As shown previously, the predominant temperature dynamics in this region are the result of periodic global heat release oscillations. High kinematic viscosity in the post-flame gases further act to dampen the turbulent fluctuations while convective and radiative heat transfer also serve to homogenize the temperature field.

[Figure 20 about here.]

\section{Conclusions}

The focus of this article was to utilize unique, new time-resolved temperature measurements (described in part 1) to improve understanding of the highly-unsteady temperature field in a turbulent swirl-flame operating with self-excited thermo-acoustic pulsations. The time-averaged temperature contours showed a very flat flame, stabilizing approximately $10 \mathrm{~mm}$ downstream of the burner face. A very high level of unsteadiness was observed in the temperature fluctuation RMS, which were $>400 \mathrm{~K}$ for $y<15 \mathrm{~mm}$ with lower fluctuation amplitudes $>100 \mathrm{~K}$ throughout the post-flame gases. The probability distribution functions exhibit a bimodal shape throughout the reaction zone, indicating that some portion of the dynamics was likely due to turbulent mixing effects, in addition to unsteady variation in the progress of chemical reactions.

Fourier analysis of the temperature measurement time series at each point in the flow revealed multiple coherent oscillatory modes. The most prevalent temperature oscillation was shown to be coherent with the combustion chamber pressure oscillations, occurring at $314 \mathrm{~Hz}$. Phase-conditioned average temperature fields elucidated an axial pumping mechanism of low-temperature reactants, which were consumed after a convective delay and resulted in a heat-release peak that was in-phase with the peak amplitude of the chamber pressure oscillation near the burner face. A power-spectral density of the temperature fluctuations relative to the phase-conditioned mean showed that a significant portion of the fluctuation energy was contained in modes other than the thermo-acoustic pulsation. Continued analysis revealed a $438 \mathrm{~Hz}$ oscillation that corresponded with the dynamics of a helical precessing vortex core. The structure of the PVC and its interaction with the flame were studied based the presence of this characteristic frequency in the power spectral densities computed throughout the flow.

The precision and time-resolution of the CPP-fsCARS measurements was sufficient to support further analysis of the underlying turbulent fluctuations. The integral time-scales were computed at each probe 
location, based on the temporal autocorrelation of the measurement time-series. PDFs of the temporal temperature gradients, normalized by $T_{A F} / t_{0}$, revealed an exponential decay for probe locations where $y \geq 15 \mathrm{~mm}$, indicating that temperature fluctuations in that region were likely due only to hydrodynamic processes. Further analysis of the state space trajectories were used to elucidate the nature of the coupling between a narrowband acoustic resonance and the highly broadband spectrum turbulent and chemical processes within the reaction zone. Computation of the largest Lyapunov spectra confirmed the presence of highly-chaotic behavior in the temperature field near the burner face and slowly decaying fluctuations in the post-flame gases.

\section{Acknowledgments}

The authors acknowledge the contributions of Michael Stöhr through complementary PIV measurements and assistance in interpretation of velocity field structure. Funding for this work was provided by the U.S. Department of Energy, Division of Chemical Sciences, Geosciences and Biosciences under Grant No. DEFG02-03ER15391 and by the King Abdullah University of Science and Technology under Award No. $1975-01$. The ultrafast laser system was purchased with funding from AFOSR DURIP Grant No. FA9550-09-1-0387.

\section{References}

[1] A. Lefebvre, Gas Turbine Combustion, Combustion: An International Series, Taylor \& Francis Group, 1999.

[2] O. Lucca-Negro, T. O'Doherty, Vortex breakdown: A review, Progress in Energy and Combustion Science 27 (2001) 431-481.

[3] N. Syred, A review of oscillation mechanisms and the role of the precessing vortex core (PVC) in swirl combustion systems, Progress in Energy and Combustion Science 32 (2006) 93-161.

[4] S. Candel, D. Durox, T. Schuller, P. Palies, J.-F. Bourgouin, J. P. Moeck, Progress and challenges in swirling flame dynamics, Comptes Rendus Mécanique 340 (2012) 758-768.

[5] T. Poinsot, D. Veynante, Theoretical and Numerical Combustion, CERFACS, third edition, 2005.

[6] T. Lieuwen, V. Yang, Combustion instabilities in gas turbine engines: operational experience, fundamental mechanisms and modeling, Progress in astronautics and aeronautics, American Institute of Aeronautics and Astronautics, 2005.

[7] R. S. Barlow, Laser diagnostics and their interplay with computations to understand turbulent combustion, Proceedings of the Combustion Institute 31 (2007) 49-75.

[8] W. P. Shih, J. G. Lee, D. a. Santavicca, Stability and emissions characteristics of a lean premixed gas turbine combustor, Proceedings of the Combustion Institute 26 (1996) 2771-2778.

[9] F. Dinkelacker, A. Soika, D. Most, D. Hofmann, a. Leipertz, W. Polifke, K. Döbbeling, Structure of locally quenched highly turbulent lean premixed flames, Proceedings of the Combustion Institute 27 (1998) 857-865.

[10] S.-Y. Lee, S. Seo, J. Broda, S. Pal, R. Santoro, An experimental estimation of mean reaction rate and flame structure during combustion instability in a lean premixed gas turbine combustor, Proceedings of the Combustion Institute 28 (2000) $775-782$.

[11] C. S. Cooper, N. M. Laurendeau, Quantitative measurements of nitric oxide in high-pressure (2-5 atm), swirl-stabilized spray flames via laser-induced fluorescence, Combustion and Flame 123 (2000) 175-188.

[12] S. R. Saretto, S. Y. Lee, J. Brumberg, C. Conrad, S. Pal, R. J. Santoro, Studies of detonation transition in a gradual area expansion for multi-cycle PDE applications, Proceedings of the Combustion Institute 30 (2005) $2809-2816$.

[13] P. Weigand, W. Meier, X. R. Duan, W. Stricker, M. Aigner, Investigations of swirl flames in a gas turbine model combustor: Flow field, structures, temperature, and species distributions, Combustion and Flame 144 (2006) $205-224$.

[14] W. Meier, X. R. Duan, P. Weigand, Investigations of swirl flames in a gas turbine model combustor: Turbulence chemistry interactions, Combustion and Flame 144 (2006) 225-236.

[15] W. Meier, I. Boxx, M. Stöhr, C. D. Carter, Laser-based investigations in gas turbine model combustors, Experiments in Fluids 49 (2010) 865-882.

[16] R. Sadanandan, M. Stöhr, W. Meier, Simultaneous OH-PLIF and PIV measurements in a gas turbine model combustor, Applied Physics B 90 (2008) 609-618.

[17] M. Stöhr, R. Sadanandan, W. Meier, Experimental study of unsteady flame structures of an oscillating swirl flame in a gas turbine model combustor, Proceedings of the Combustion Institute 32 (2009) 2925-2932. 
[18] C. Fajardo, J. Smith, V. Sick, Sustained simultaneous high-speed imaging of scalar and velocity fields using a single laser, Applied Physics B 85 (2006) 25-30.

[19] I. Boxx, M. Stöhr, C. Carter, W. Meier, Sustained multi-kHz flamefront and 3-component velocity-field measurements for the study of turbulent flames, Applied Physics B 95 (2009) 23-29.

[20] G. Hartung, J. Hult, R. Balachandran, M. R. Mackley, C. F. Kaminski, Flame front tracking in turbulent lean premixed flames using stereo PIV and time-sequenced planar LIF of OH, Applied Physics B 96 (2009) 843-862.

[21] I. Boxx, M. Stöhr, C. Carter, W. Meier, Temporally resolved planar measurements of transient phenomena in a partially pre-mixed swirl flame in a gas turbine model combustor, Combustion and Flame 157 (2010) 1510-1525.

[22] B. Böhm, C. Heeger, R. L. Gordon, A. Dreizler, New Perspectives on Turbulent Combustion: Multi-Parameter High-Speed Planar Laser Diagnostics, Flow, Turbulence and Combustion 86 (2010) 313-341.

[23] C. Abram, B. Fond, A. L. Heyes, F. Beyrau, High-speed planar thermometry and velocimetry using thermographic phosphor particles, Applied Physics B 111 (2013) 155-160.

[24] A. M. Steinberg, I. Boxx, M. Stöhr, C. D. Carter, W. Meier, Flow-flame interactions causing acoustically coupled heat release fluctuations in a thermo-acoustically unstable gas turbine model combustor, Combustion and Flame 157 (2010) $2250-2266$.

[25] M. Stöhr, R. Sadanandan, W. Meier, Phase-resolved characterization of vortex-flame interaction in a turbulent swirl flame, Experiments in Fluids 51 (2011) 1153-1167.

[26] M. Stöhr, I. Boxx, C. Carter, W. Meier, Dynamics of lean blowout of a swirl-stabilized flame in a gas turbine model combustor, Proceedings of the Combustion Institute 33 (2011) 2953-2960.

[27] M. Stöhr, I. Boxx, C. D. Carter, W. Meier, Experimental study of vortex-flame interaction in a gas turbine model combustor, Combustion and Flame 159 (2012) 2636-2649.

[28] M. Stöhr, C. Arndt, W. Meier, Effects of Damköhler number on vortex-flame interaction in a gas turbine model combustor, Proceedings of the Combustion Institute 34 (2013) 3107-3115.

[29] A. Eckbreth, Laser Diagnostics for Combustion Temperature and Species, Taylor \& Francis, second edition, 1996.

[30] J. J. Barrett, Generation of coherent anti-Stokes rotational Raman radiation in hydrogen gas, Applied Physics Letters 29 (1976) $722-724$.

[31] A. Eckbreth, T. Anderson, Dual broadband CARS for simultaneous, multiple species measurements, Applied Optics 24 (1985) 2731-2736.

[32] R. P. Lucht, R. E. Palmer, M. A. Maris, Simultaneous acquisition of pure rotational and vibrational nitrogen spectra using three-laser coherent anti-Stokes Raman spectroscopy, Optics Letters 12 (1987) 386-388.

[33] P. E. Bengtsson, L. Martinsson, M. Alden, Combined Vibrational and Rotational CARS for Simultaneous Measurements of Temperature and Concentrations of Fuel, Oxygen, and Nitrogen, Journal of Applied Spectroscopy 49 (1995) 188-192.

[34] C. Brackmann, J. Bood, P. Bengtsson, T. Seeger, M. Schenk, A. Leipertz, Combined Vibrational and Rotational CARS for Simultaneous Measurements of Temperature and Concentrations of Fuel, Oxygen, and Nitrogen, Journal of Applied Optics 41 (2002) 564-572.

[35] T. Seeger, F. Beyrau, A. Brauer, A. Leipertz, High-pressure pure rotational CARS: comparison of temperature measurements with O2, N2, and synthetic air, Journal of Raman Spectroscopy 34 (2003) 932-939.

[36] S. Roy, T. R. Meyer, R. P. Lucht, M. Afzelius, P.-E. Bengtsson, J. R. Gord, Dual-pump dual-broadband coherent antiStokes Raman scattering in reacting flows, Optics Letters 29 (2004) 1843-1845.

[37] T. R. Meyer, S. Roy, R. P. Lucht, J. R. Gord, Dual-pump dual-broadband CARS for exhaust-gas temperature and CO2-O2-N2 mole-fraction measurements in model gas-turbine combustors, Combustion and Flame 142 (2005) 52-61.

[38] M. Thariyan, V. Ananthanarayanan, A. Bhuiyan, S. Naik, J. Gore, R. Lucht, Dual-pump CARS temperature and major species concentration measurements in counter-flow methane flames using narrowband pump and broadband Stokes lasers, Combustion and Flame (2010).

[39] A. Satija, R. P. Lucht, Development of a combined pure rotational and vibrational coherent anti-Stokes Raman scattering system., Optics letters 38 (2013) 1340-2.

[40] R. P. Lucht, S. Roy, T. R. Meyer, J. R. Gord, Femtosecond coherent anti-Stokes Raman scattering measurement of gas temperatures from frequency-spread dephasing of the Raman coherence, Applied Physics Letters 89 (2006) 251112.

[41] R. P. Lucht, P. J. Kinnius, S. Roy, J. R. Gord, Theory of femtosecond coherent anti-Stokes Raman scattering spectroscopy of gas-phase transitions., The Journal of Chemical Physics 127 (2007) 044316.

[42] J. R. Gord, T. R. Meyer, S. Roy, Applications of ultrafast lasers for optical measurements in combusting flows., Annual review of analytical chemistry 1 (2008) 663-87.

[43] S. Roy, P. J. Kinnius, R. P. Lucht, J. R. Gord, Temperature measurements in reacting flows by time-resolved femtosecond coherent anti-Stokes Raman scattering (fs-CARS) spectroscopy, Optics Communications 281 (2008) 319-325.

[44] S. Roy, J. R. Gord, A. K. Patnaik, Recent advances in coherent anti-Stokes Raman scattering spectroscopy: Fundamental developments and applications in reacting flows, Progress in Energy and Combustion Science 36 (2010) 280-306.

[45] D. R. Richardson, R. P. Lucht, W. D. Kulatilaka, S. Roy, J. R. Gord, Theoretical modeling of single-laser-shot, chirpedprobe-pulse femtosecond coherent anti-Stokes Raman scattering thermometry, Applied Physics B 104 (2011) 699-714.

[46] D. R. Richardson, R. P. Lucht, S. Roy, W. D. Kulatilaka, J. R. Gord, Single-laser-shot femtosecond coherent anti-Stokes Raman scattering thermometry at $1000 \mathrm{~Hz}$ in unsteady flames, Proceedings of the Combustion Institute 33 (2011) 839-845.

[47] D. R. Richardson, D. Bangar, R. P. Lucht, Polarization suppression of the nonresonant background in femtosecond coherent anti-Stokes Raman scattering for flame thermometry at 5 kHz., Optics express 20 (2012) 21495-504.

[48] C. N. Dennis, C. D. Slabaugh, I. G. Boxx, W. Meier, R. P. Lucht, Chirped probe pulse femtosecond coherent anti-Stokes Raman scattering thermometry at $5 \mathrm{kHz}$ in a Gas Turbine Model Combustor, Proceedings of the Combustion Institute (2014). 
[49] C. N. Dennis, C. D. Slabaugh, I. G. Boxx, W. Meier, R. P. Lucht, 5 kHz Thermometry in a Swirl-Stabilized Gas Turbine Model Combustor using Chirped Probe Pulse Femtosecond CARS.

Part 1: Experimental Measurements, Combustion and Flame (????).

[50] A. Oppenheim, R. Schafer, Digital Signal Processing, Prentice-Hall, 2011.

[51] A. M. Steinberg, I. Boxx, M. Stöhr, W. Meier, C. D. Carter, Effects of Flow Structure Dynamics on Thermoacoustic Instabilities in Swirl-Stabilized Combustion, AIAA Journal 50 (2012) 952-967.

[52] A. M. Gurvich, A. S. nd Yaglom, Breakdown of Eddies and Probability Distributions for Small Scale Turbulence, Physics of Fluids 10 (1967) 59-65.

[53] G.-H. Wang, N. Clemens, P. Varghese, High-repetition rate measurements of temperature and thermal dissipation in a non-premixed turbulent jet flame, Proceedings of the Combustion Institute 30 (2005) 691-699.

[54] J. Sprott, Chaos and Time-series Analysis, Oxford University Press, 2003.

[55] G. Haller, T. Sapsis, Lagrangian coherent structures and the smallest finite-time Lyapunov exponent., Chaos 21 (2011).

[56] A. Wolf, J. B. Swift, H. L. Swinney, J. a. Vastano, Determining Lyapunov exponents from a time series, Physica D: Nonlinear Phenomena 16 (1985) 285-317.

[57] M. Rosenstein, J. Collins, C. de Luca, A practical method for calculating largest Lyapunov exponents from small data sets, Physica D-Nonlinear Phenomena (1993).

[58] S. L. Brunton, C. W. Rowley, Fast computation of finite-time Lyapunov exponent fields for unsteady flows., Chaos 20 (2010).

[59] J. Doyne Farmer, Chaotic attractors of an infinite-dimensional dynamical system, Physica D: Nonlinear Phenomena 4 (1982) 366-393.

[60] G. Xiaofeng, C. H. Lai, Detecting chaos from time series, Journal of Physics A: Mathematical and General 33 (2000) $1007-1016$.

[61] S. Narayanan, F. Hussain, Measurements of spatiotemporal dynamics in a forced plane mixing layer, Journal of Fluid Mechanics 320 (1996).

[62] G. Broze, F. Hussain, Nonlinear dynamics of forced transitional jets: periodic and chaotic attractors, Journal of Fluid Mechanics 263 (1994) 93.

[63] A. C. Noble, G. B. King, N. M. Laurendeau, J. R. Gord, S. Roy, Nonlinear Thermoacoustic Instability Dynamics in a Rijke Tube, Combustion Science and Technology 184 (2012) 293-322.

[64] T. Sapsis, G. Haller, Clustering criterion for inertial particles in two-dimensional time-periodic and three-dimensional steady flows., Chaos 20 (2010) 017515. 


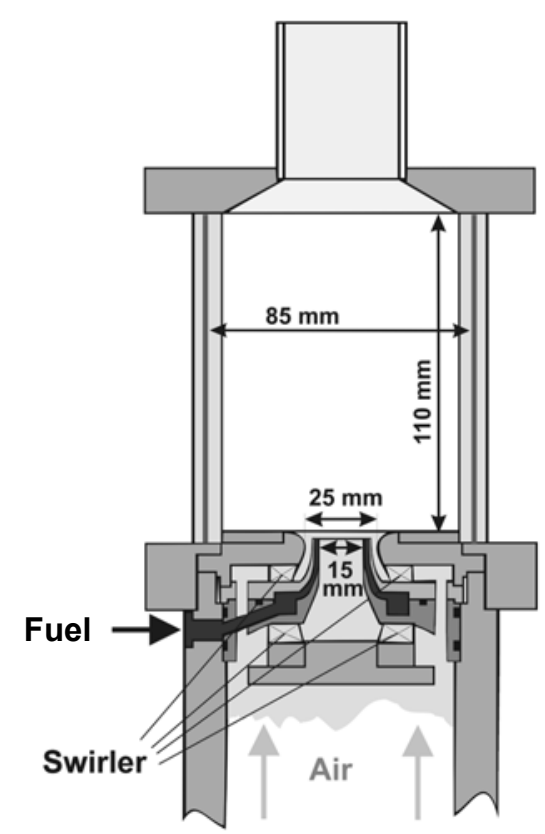

Figure 1: Schematic diagram of the Dual-Swirl GTMC. 


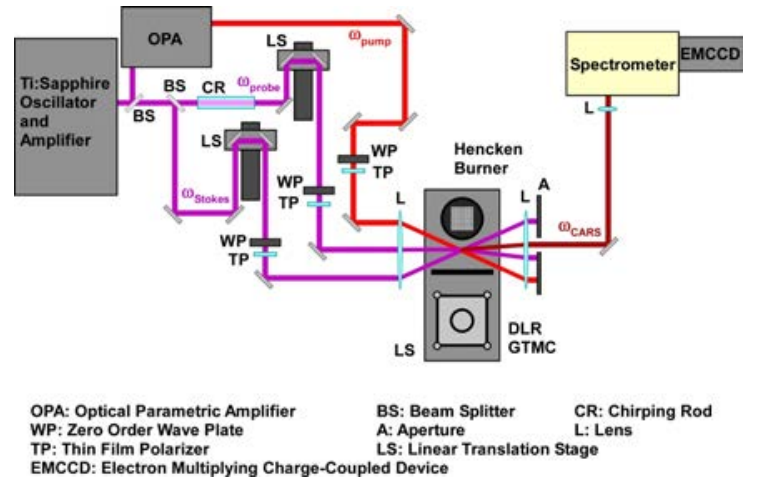

Figure 2: Chirped probe pulse femtosecond CARS measurement system. 

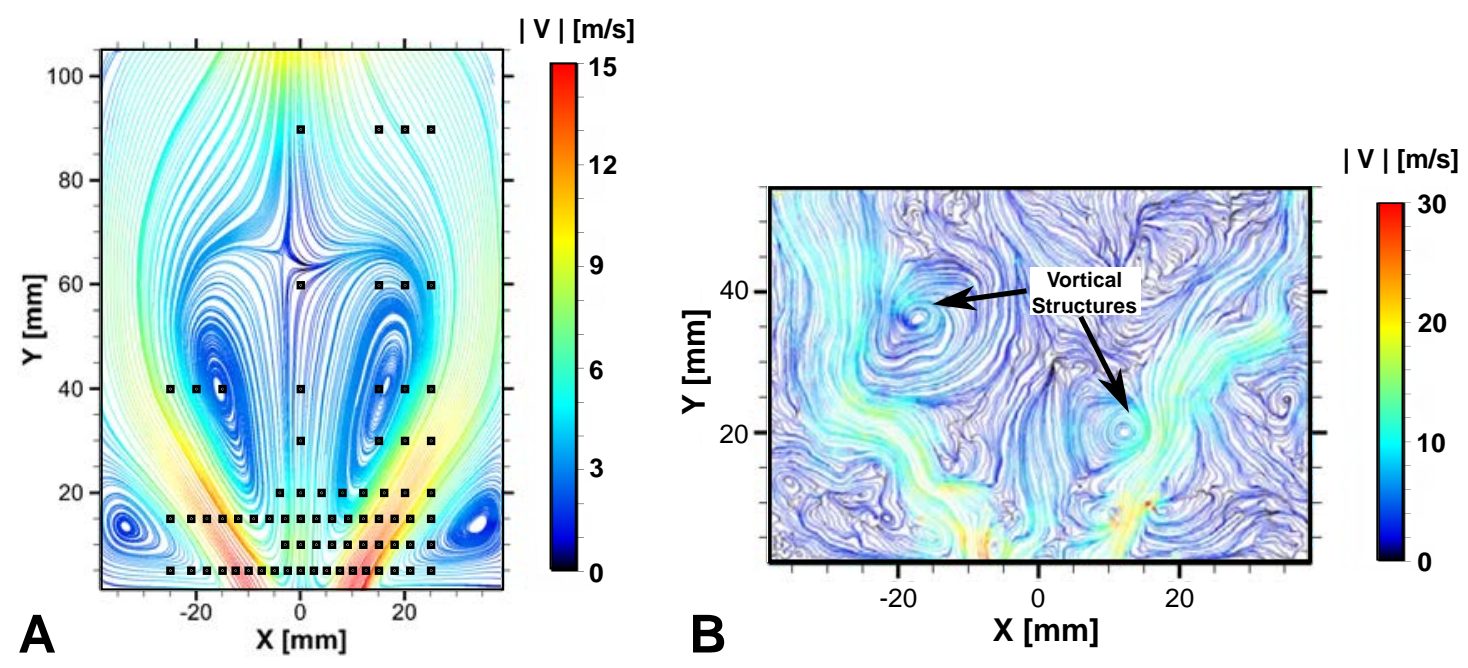

Figure 3: Flame B velocity fields measured with PIV [16, 27]: CPP-fsCARS measurement probe locations overlaid against mean velocity field (A) and typical instantaneous flow field showing evidence of helical precessing vortex core (B). 


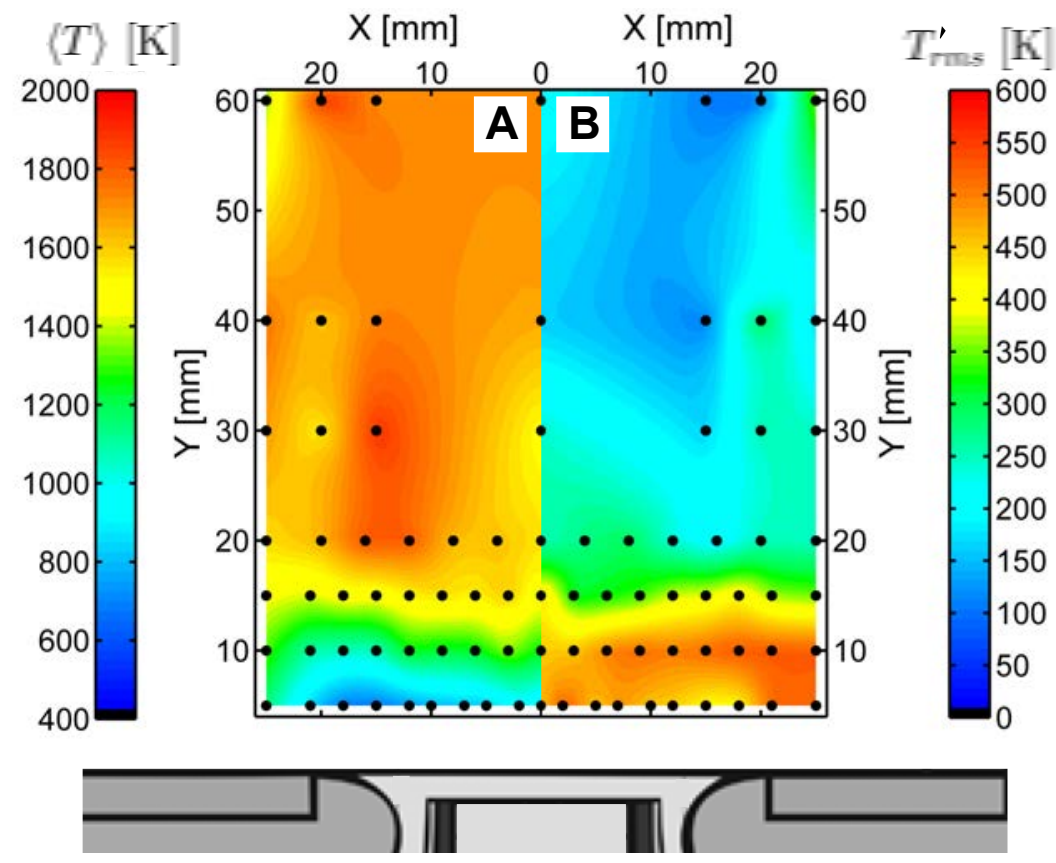

Figure 4: Time-averaged temperature field (A) and temperature fluctuation RMS field (B). 

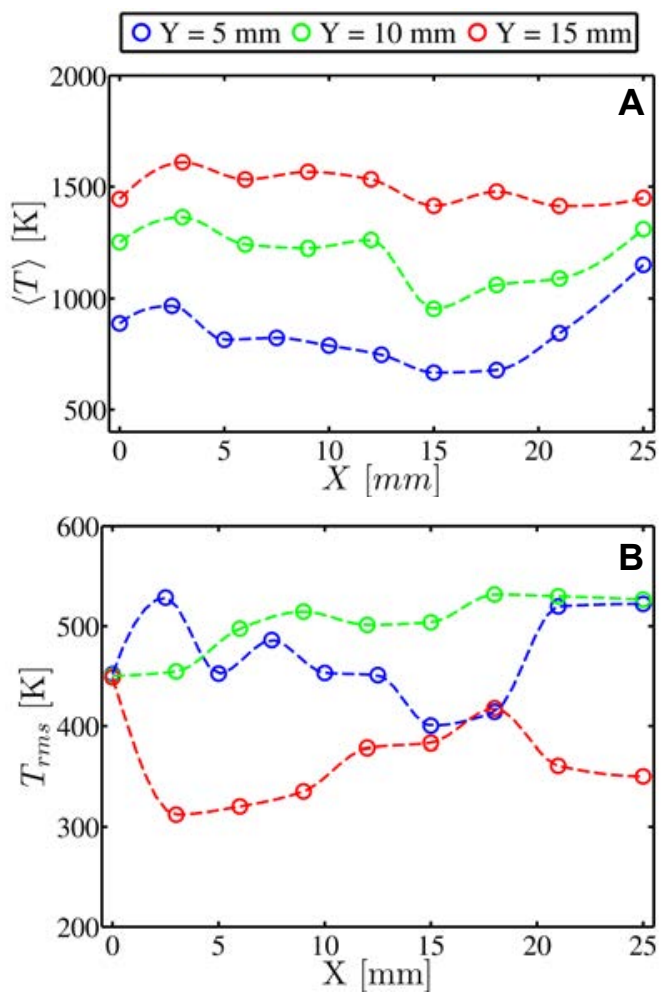

Figure 5: Radial profiles of the temperature time-average (A) and fluctuation RMS (B). 

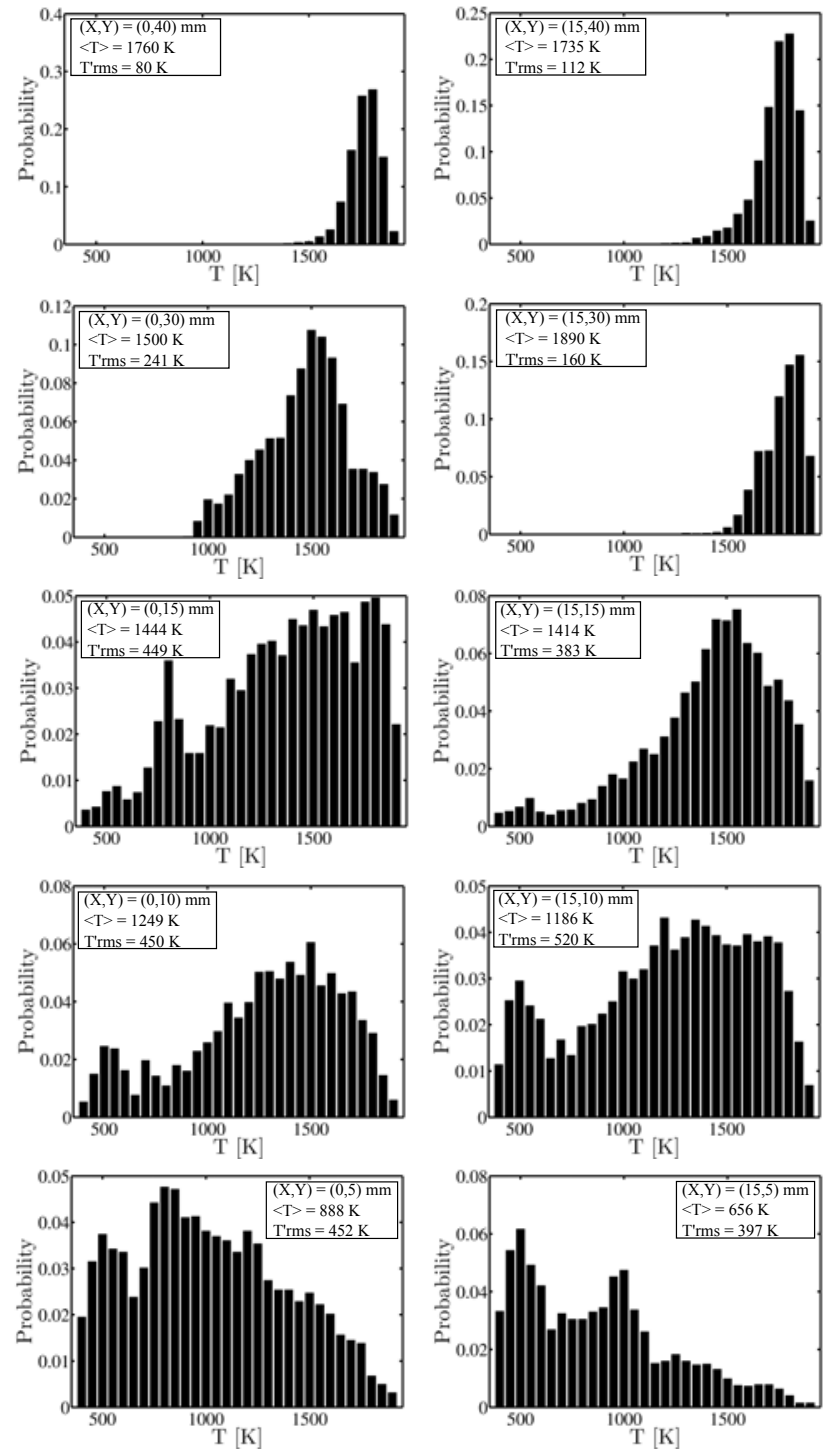

Figure 6: Probability density functions computed from single-shot CPP-fsCARS temperature measurements through the reaction zone and in the post-flame region. 
1

2

3

4

5

6

7

8

9

10

11

12

13

14

15

16

17

18

19

20

21

22

23

24

25

26

27

28

29

30

31

32

33

34

35

36

37

38

39

40

41

42

43

44

45

46

47

48

49

50

51

52

53

54

55

56

57

58

59

60

61

62

63

64

65

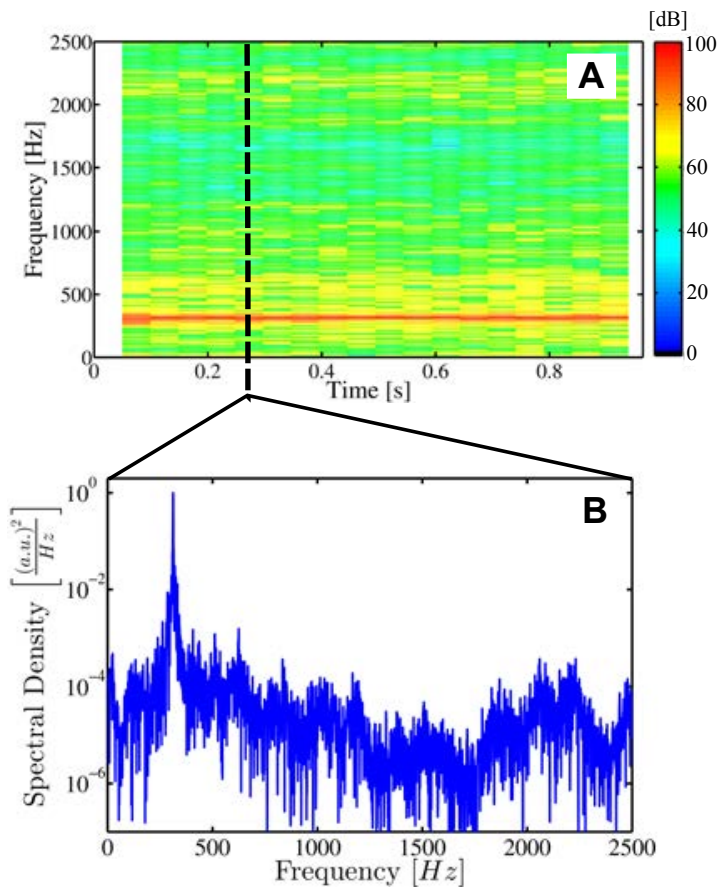

Figure 7: Typical spectrogram from an acoustic measurement time series (A) and the peak-normalized power spectra computed from plenum microphone signal (B). 

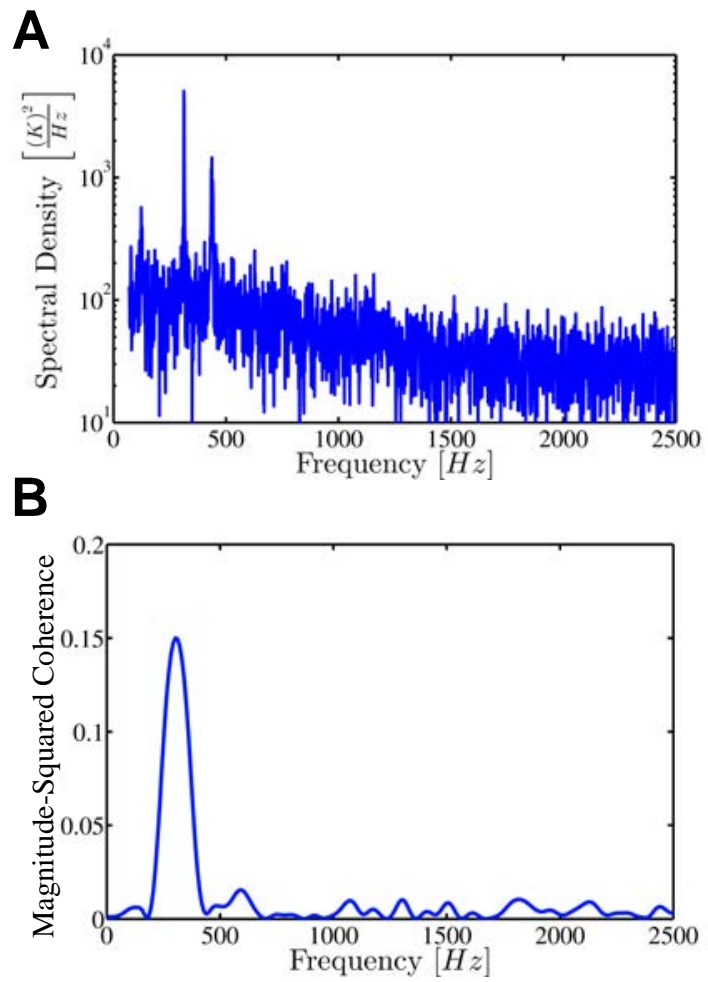

Figure 8: Power spectrum of the temperature fluctuation at $(\mathrm{x}, \mathrm{y})=(15,5) \mathrm{mm}(\mathrm{A})$ with corresponding cross spectral density illustrating signal coherence between temperature and pressure fluctuations at $314 \mathrm{~Hz}(\mathrm{~B})$. 
1

2

3

4

5

6

7

9

10

11

12

13

14

15

16

17

18

19

20

21

22

23

24

25

26

27

28

29

30

31

32

33

34

35

36

37

38

39

40

41

42

43

44

45

46

47

48

49

50

51

52

53

54

55

56

57

58

59

60

61

62

63

64

65

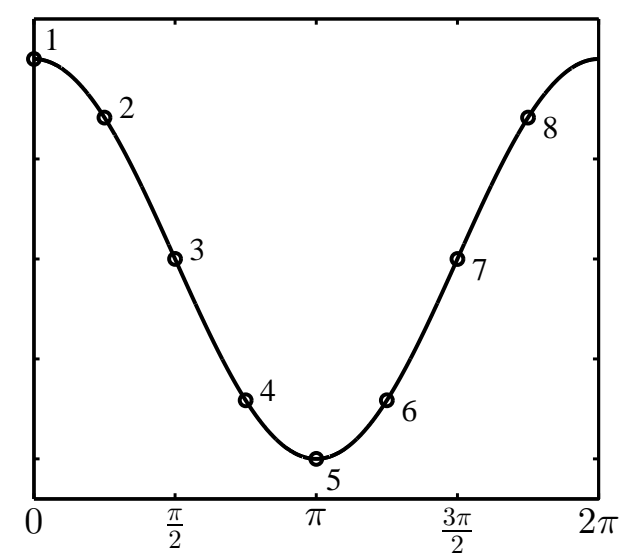

Figure 9: Phase angle assignment for phase conditioned statistical analyses. 


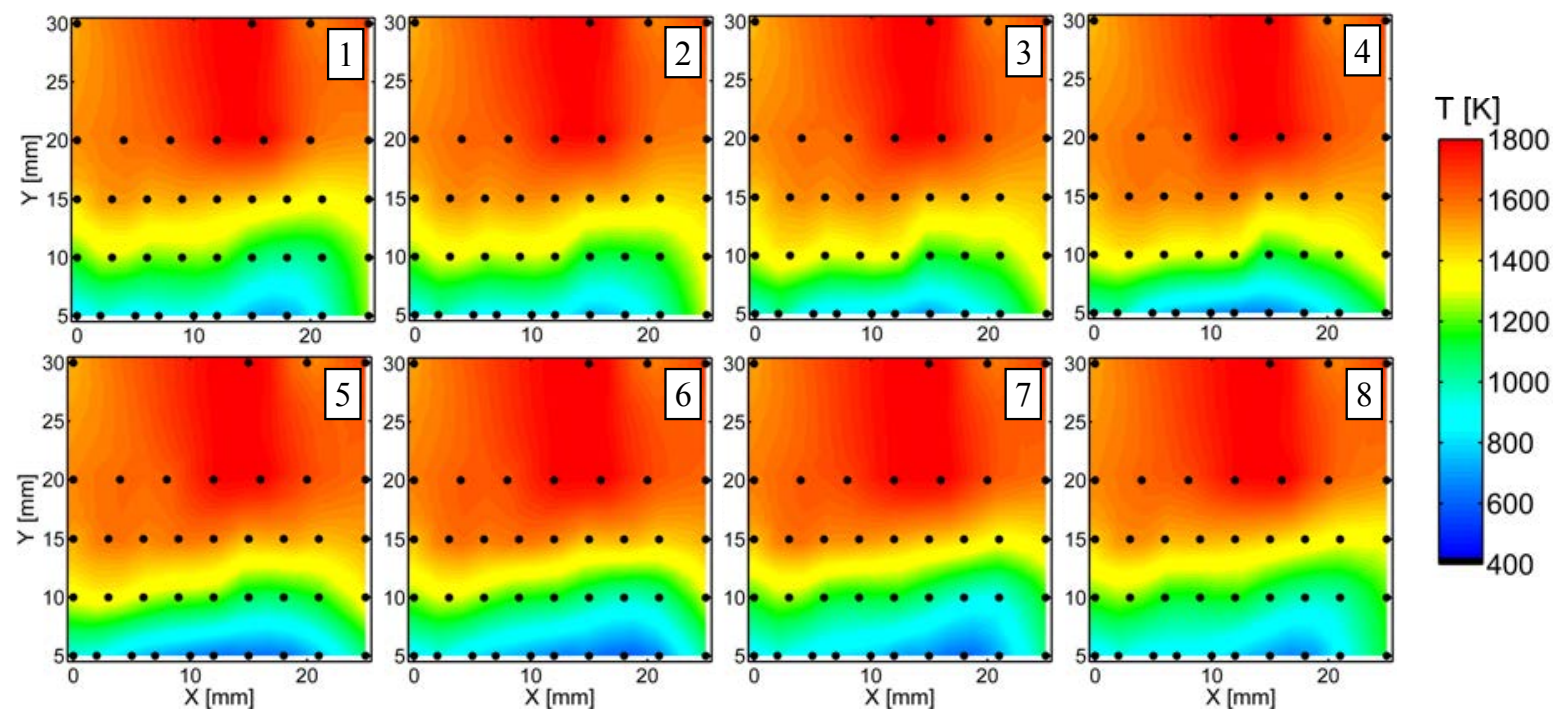

Figure 10: Phase-conditioned average temperature fields. 

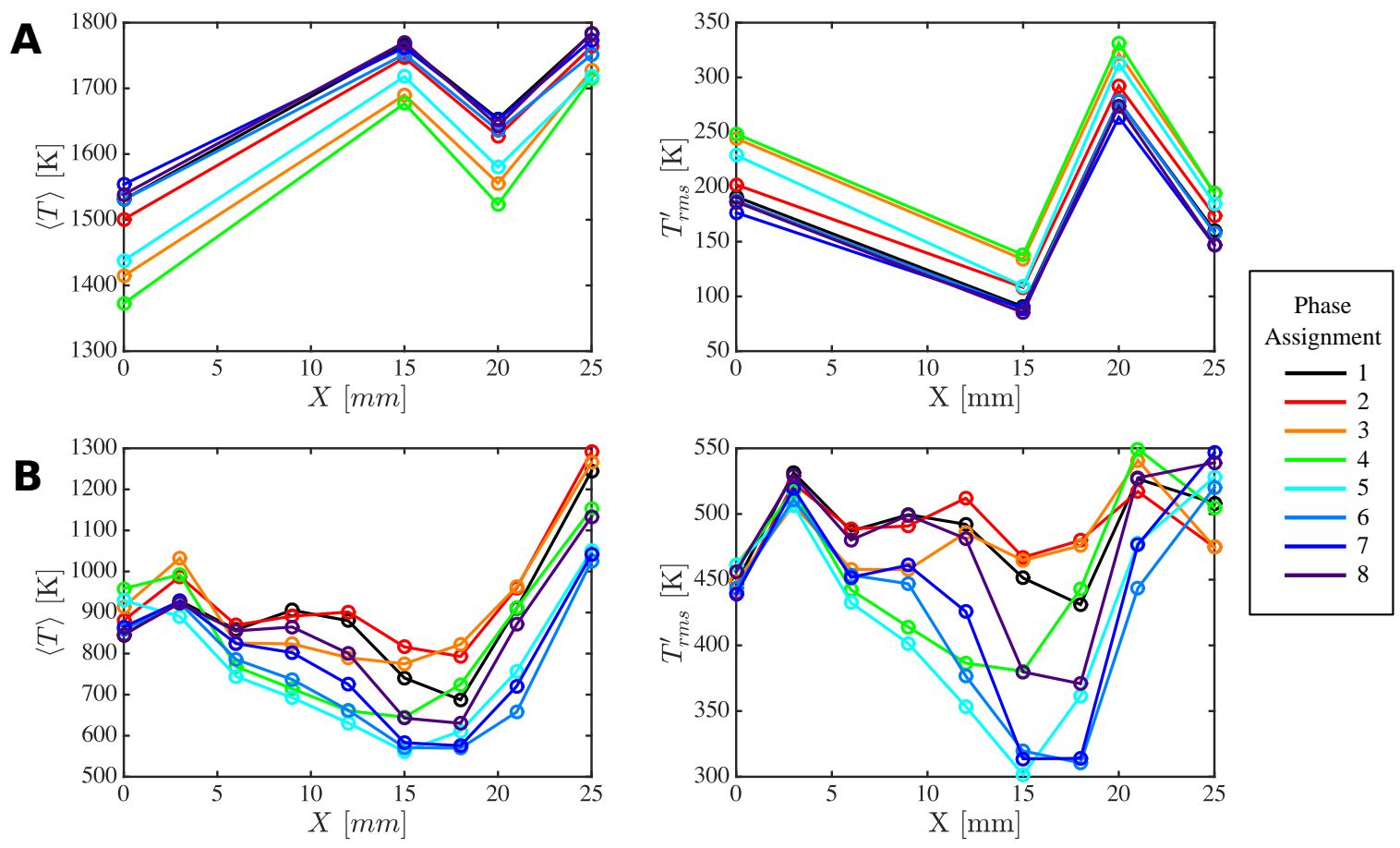

Figure 11: Phase-conditioned radial profiles of average temperature (left column) and temperature fluctuation RMS (right column) for two burner heights: $y=30 \mathrm{~mm}$ (A) and $y=5 \mathrm{~mm}$ (B). 


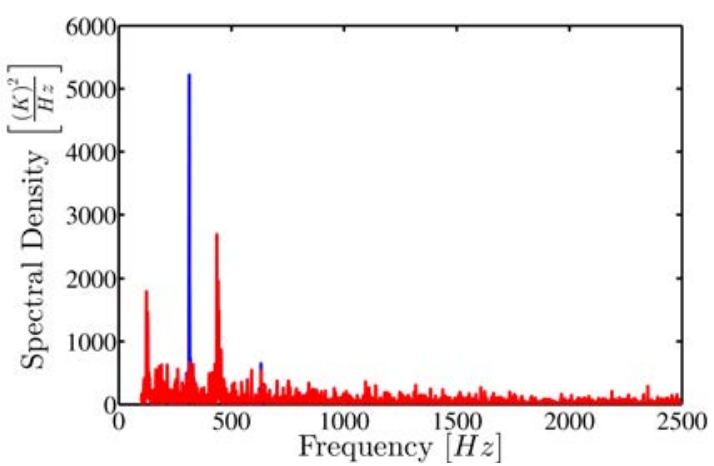

Figure 12: Power spectrum of the temperature fluctuation at $(\mathrm{x}, \mathrm{y})=(15,5) \mathrm{mm}$ relative to the long-time mean (blue) and the phase-conditioned mean (red). 

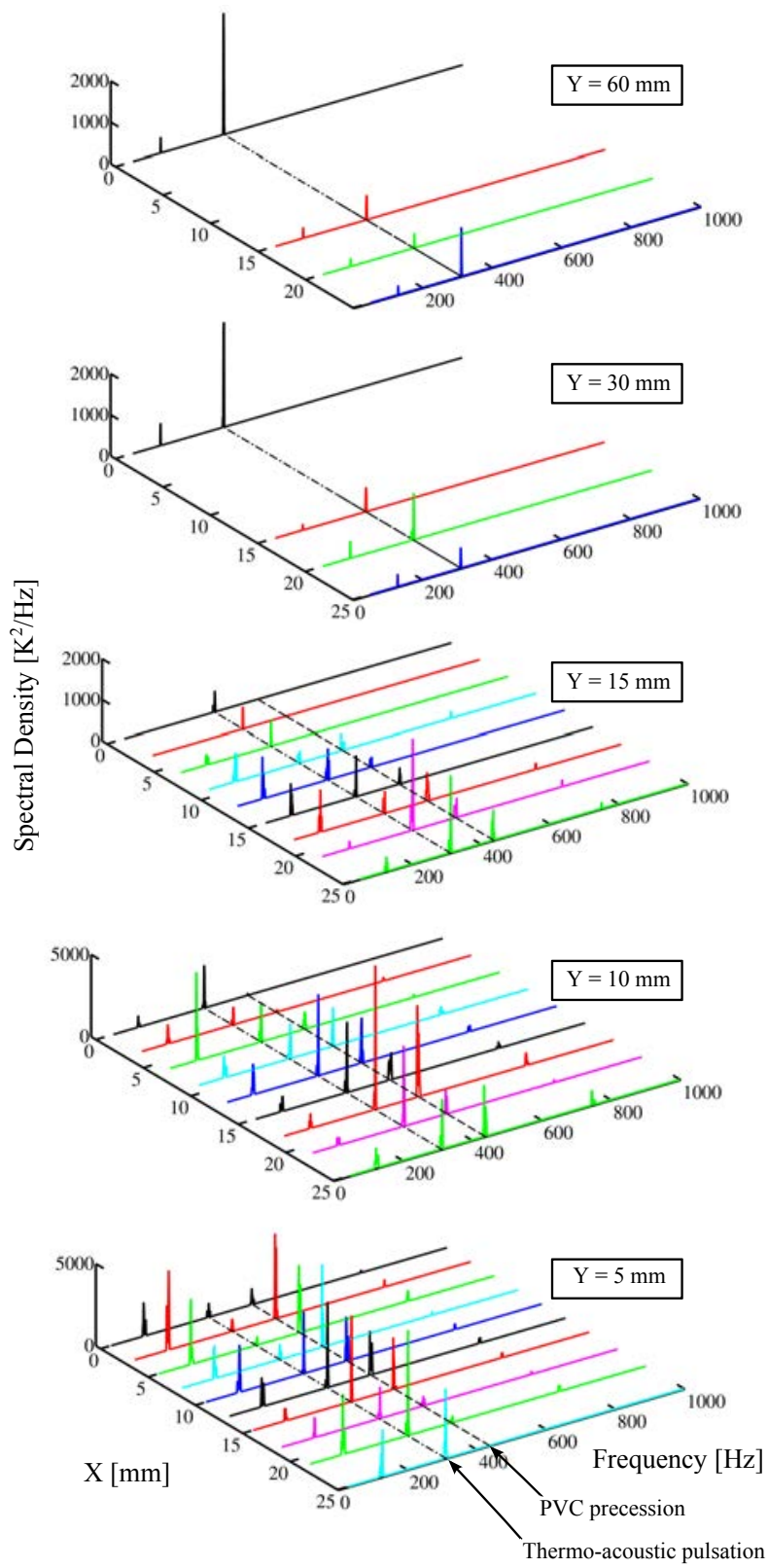

Figure 13: Power spectra plotted as a function of burner radius at varying heights to show spatial variation of frequency content within the flow. 
1

2

3

4

5

6

7

8

10

11

12

13

14

15

16

17

18

19

20

21

22

23

24

25

26

27

28

29

30

31

32

33

34

35

36

37

38

39

40

41

42

43

44

45

46

47

48

49

50

51

52

53

54

55

56

57

58

59

60

61

62

63

64

65

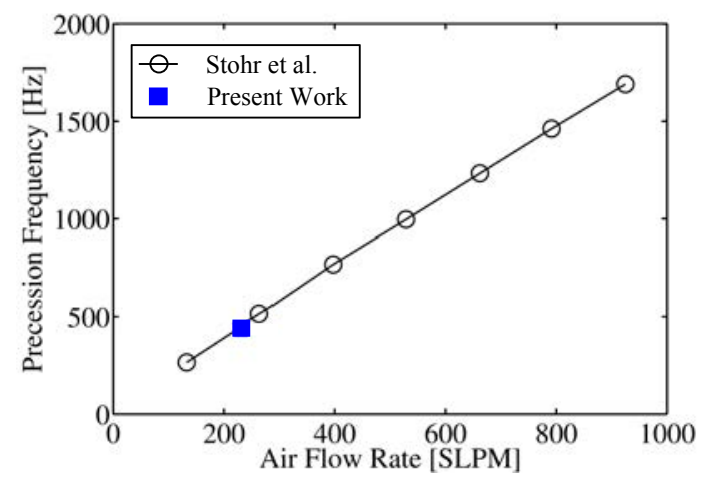

Figure 14: PVC precession frequency as a function of air flow rate (adapted from data presented in Stohr et al. [27]). 

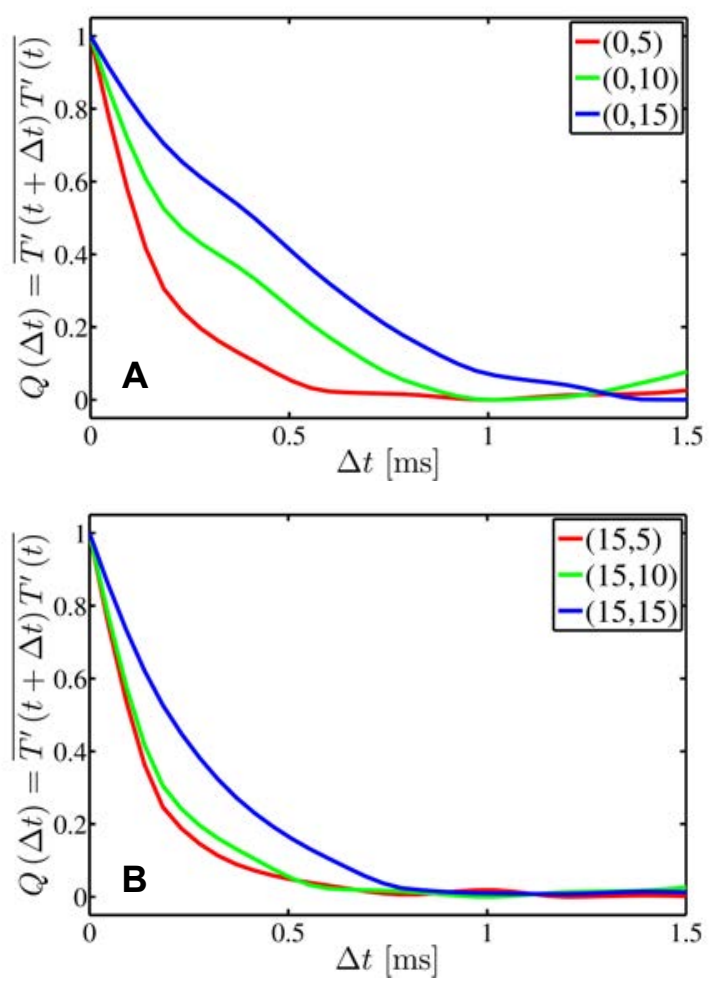

Figure 15: Temporal autocorrelation of the temperature fluctuation time series measurements at three heights along the channel centerline (A) and through the inner shear layer (B). 
1

2

3

4

5

6

7

8

9

10

11

12

13

14

15

16

17

18

19

20

21

22

23

24

25

26

27

28

29

30

31

32

33

34

35

36

37

38

39

40

41

42

43

44

45

46

47

48

49

50

51

52

53

54

55

56

57

58

59

60

61

62

63

64

65

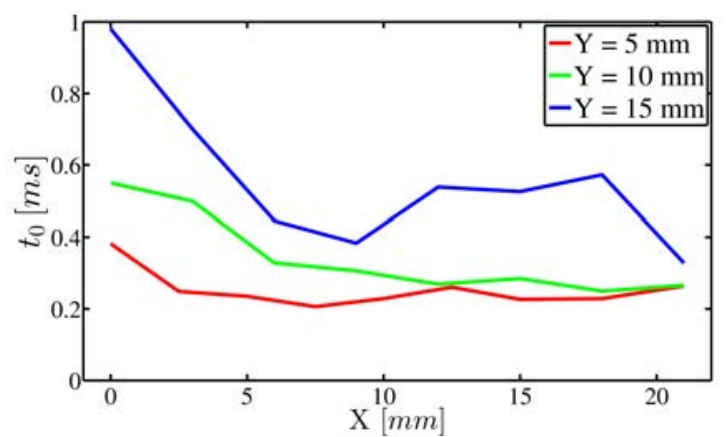

Figure 16: Radial profiles of the integral time-scales computed from the temperature fluctuation time series at three probe heights within the reaction zone. 

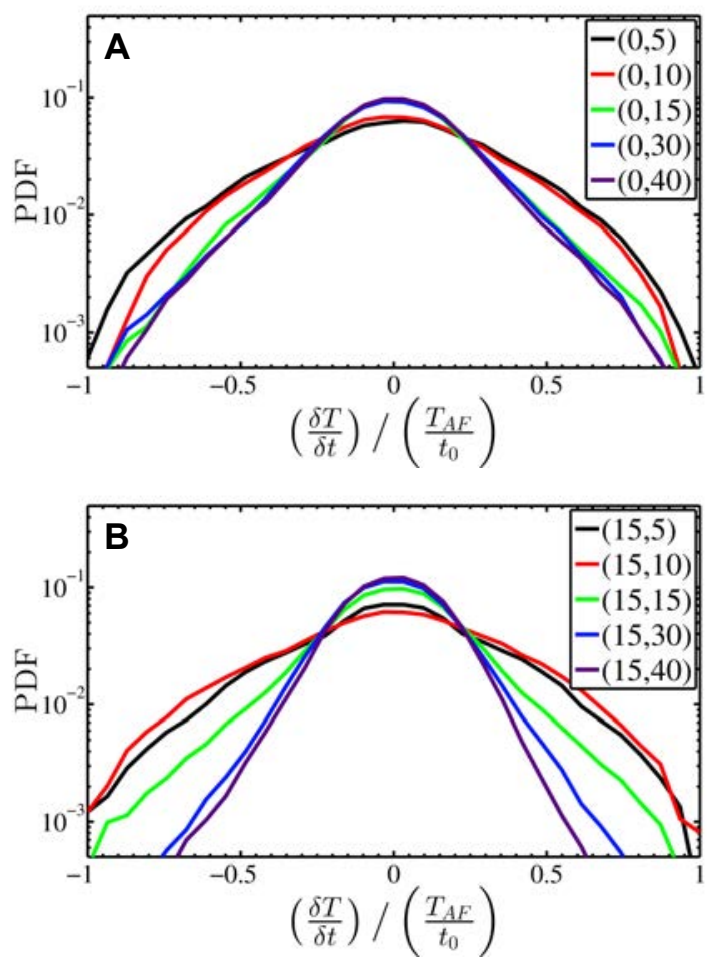

Figure 17: Probability distribution functions of the $\delta T / \delta t$, normalized by the adiabatic flame temperature $\left(T_{A F}\right)$ and the integral time scale $\left(t_{0}\right)$ computed from the time-resolved measurements at that probe location. 


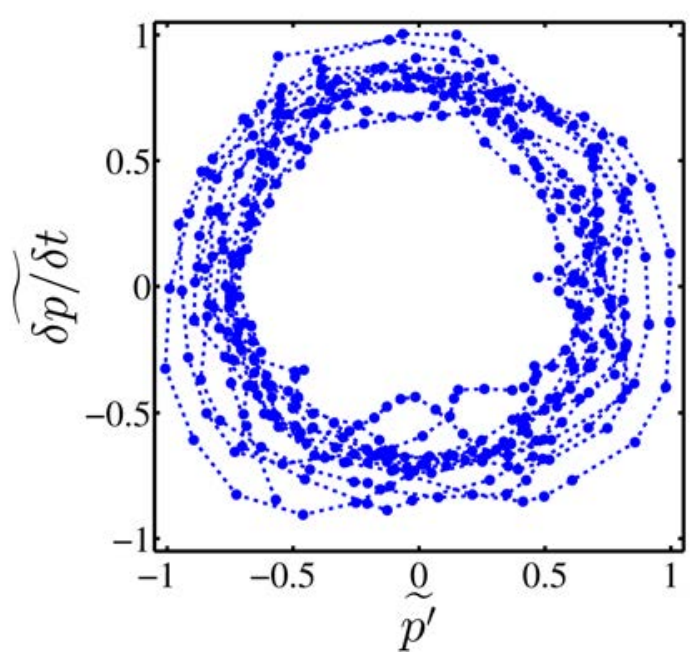

Figure 18: The state diagram of $p^{\prime}$ vs. $\delta p / \delta t$ exhibits the coherent dynamic behavior of the combustion chamber acoustics. 

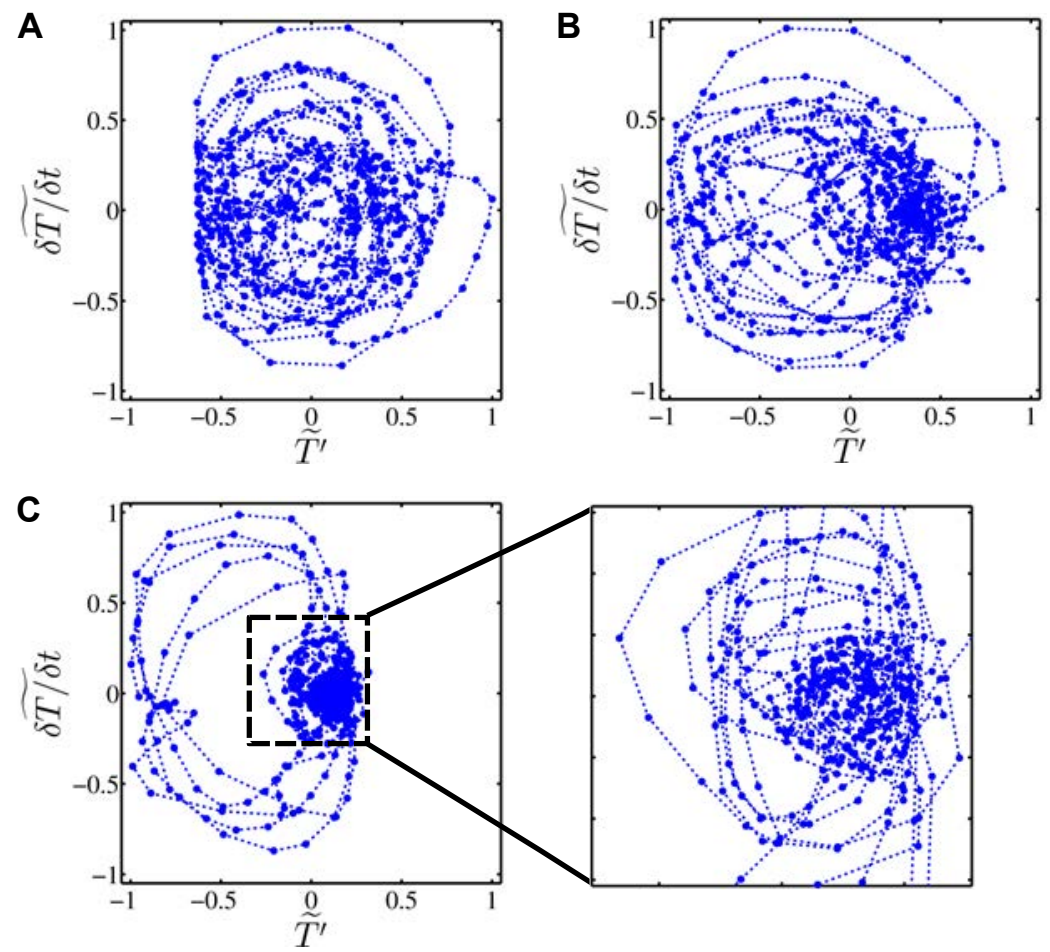

Figure 19: The state space of $T^{\prime}$ vs. $\delta T / \delta t$ at $(x, y)=(15,5) m m(\mathrm{~A}),(x, y)=(15,15) m m(\mathrm{~B})$, and $(x, y)=(15,30) m m(\mathrm{C})$. 


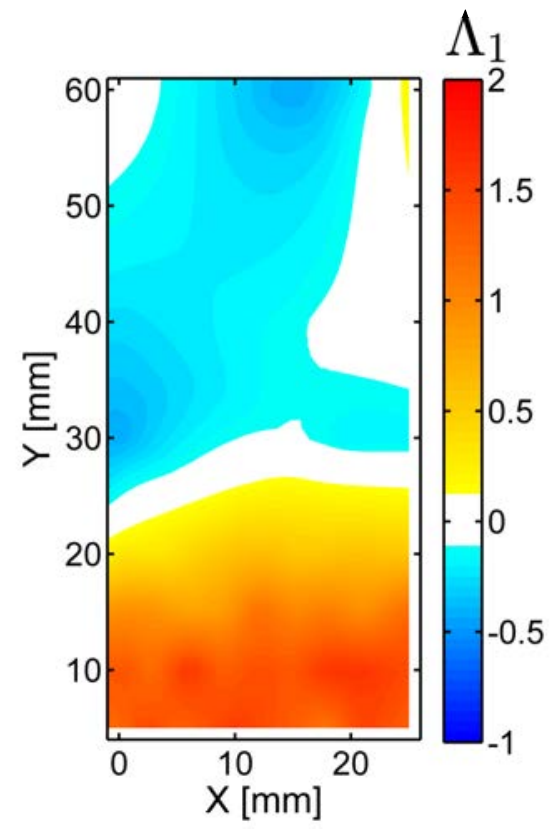

Figure 20: Contour map of the first Lyapunov exponent field. 